\title{
（152） QUICK STRUCTURAL DAMAGE ESTIMATION BY NEURAL NETWORKS
}

\author{
Gilbert L. MOLAS \\ Fumio YAMAZAKI
}

\author{
Graduate Student, University of Tokyo \\ Associate Professor, University of Tokyo
}

\section{INTRODUCTION}

When a strong earthquake occurs in a large city, it is imperative to assess the damage quickly. A quick assessment alerts emergency service agencies. It also gives city gas companies valuable information to decide whether to cut off the gas supply to avoid secondary disasters like the spread of fire. Neural networks present a new approach to obtaining quick assessment. Neural networks have been found to be an excellent technique in finding a relationship between the input and output values even though that relationship is complex and unknown. Neural networks have been used recently in the field of earthquake engineering $[1,2]$ because of its versatility and robustness in seeking complex relationships by itself. However, the effectiveness of neural networks depends largely on the "quality" of the data to be used for training the network. Since the number of data available for training the network is still quite small, the network may not be able to predict "correctly" for certain cases. To overcome this problem, this study uses simulated data so that the network may be trained for the whole range of expected values that will be handled.

\section{SIMULATION OF STRONG GROUND MOTION}

In order not to introduce too much complexity at this time, the Kanai-Tajimi (K-T) power spectra representation is used in this study because of its simplicity. Lai [3] has studied the statistical characteristics (e.g., mean and coefficient of variation) of the K-T parameters based on actual earthquakes. The K-T parameters that need to be specified are the intensity, $S_{0}$, the K-T frequency, $\omega_{g}$, and the K-T damping, $h_{g}$. In this study, the values of the parameters determined by Lai [3] are used as typical values. To complete the description of the simulated earthquake, a trapezoidal envelope function with a $2.5 \mathrm{~s}$ rise and decay time is used, again for simplicity. To have a well-distributed set of strong ground motion data, the parameters for the simulation are randomly selected from the range of values given in Table 1, assuming a uniform distribution for the parameters. To have a well-trained network that can generalize well, a large number of training data must be used. In this study, 500 ground motion time series are generated. Figure 1 shows the distribution of the maximum acceleration and velocity for the set of simulated earthquakes.

\section{STRUCTURE / DAMAGE MODELS}

To estimate the damage due to strong ground motion, a step-by-step nonlinear analysis procedure is applied using two single-degree-of-freedom models representing two types of wooden framed structures commonly found in Japan. The models have bilinear stiffness with the secondary stiffness taken as $20 \%$ of the initial stiffness. The damping ratio is taken to be 0.05 and the restoring force at yielding is

$$
Q_{y}=m g \cdot C_{y} \quad \text { where } C_{y}=0.25 / \sqrt{T} .
$$

The first model (Wooden 1 ) represents ordinary wooden framed houses with two stories and a fundamental period of $T=0.55 \mathrm{~s}$. The second model (Wooden 2 ) represents fire-resisting wooden framed houses with two stories and a fundamental period of $T=0.35 \mathrm{~s}$. The damage to the structures is then given in terms of the ductility factor.

\section{NEURAL NETWORK MODEL}

The main objective in the construction of the neural network model is to estimate the damage of the structure (in terms of the ductility factor) from indices of the strong

Table 1. Parameters for earthquake simulation

\begin{tabular}{c|l|c}
\hline Parameter & Lower Limit & Upper Limit \\
\hline total time & $7.5 \mathrm{~s}$ & $20.0 \mathrm{~s}$ \\
$S_{o}$ & $1.0 \mathrm{~cm}^{2} / \mathrm{s}^{3}$ & $500.0 \mathrm{~cm}^{2} / \mathrm{s}^{3}$ \\
$\omega_{g}$ & $4.0 \mathrm{rad} / \mathrm{s}$ & $40.0 \mathrm{rad} / \mathrm{s}$ \\
& $(0.64 \mathrm{~Hz})$ & $(6.34 \mathrm{~Hz})$ \\
$h_{g}$ & 0.15 & 0.60 \\
\hline
\end{tabular}

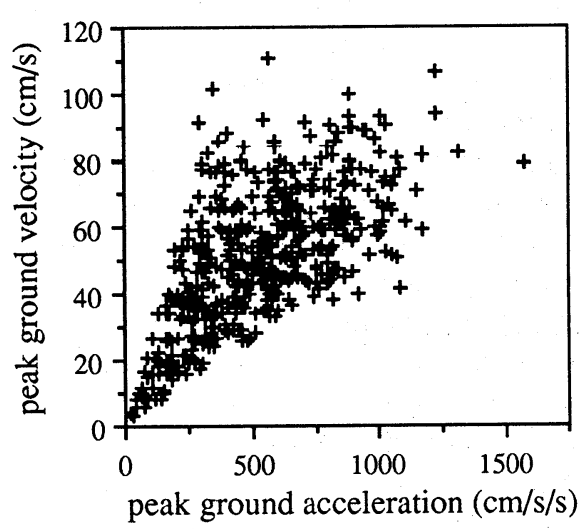

Figure 1. Distribution of peak ground acceleration and velocity for the simulated earthquakes 
ground motion only. Therefore, in an earthquake, sensors can measure the indices of the ground motion and then the neural network can immediately generate a damage estimate.

The indices used as input to the network should be simple and easy to obtain. The indices should also identify the characteristics of the ground motion in a general way. In this study, the indices include the peak ground acceleration (PGA), peak ground velocity (PGV), and peak ground displacement (PGD). The Spectral Intensity (SI) is considered because it was found to be a good index of damage and because its value can be immediately estimated using a new type of sensor [4]. To

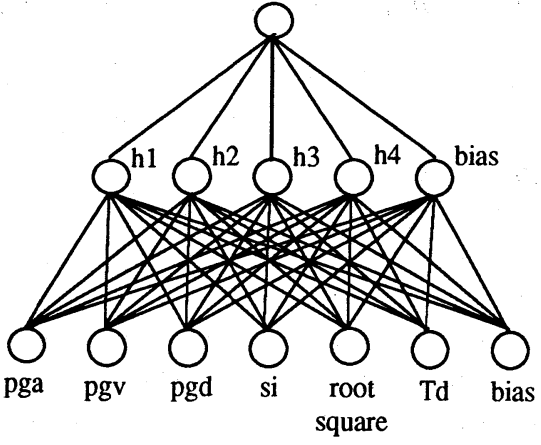

Figure 2. Neural network structure account for the averaged "intensity" of the ground motion the root square of the acceleration is used. Finally, the time duration of the ground motion, $T_{d}$, as defined by Trifunac and Brady [5] is used.

The neural network model used is a feed-forward back-propagation network having one hidden layer with four processing elements (PEs) fully connected to the input and output layers and bias (Figure 2). Two logistic activation functions, namely the sigmoid and the hyperbolic tangent functions, and three learning rules are tried and compared. These are the: 1) Normalized Cumulative Delta Rule (NCDR) [6]; 2) Extended Delta-Bar-Delta Rule (EDBD) [7]; and 3) Directed Random Search (DRS) [8]. Strictly speaking, DRS is not a back propagation algorithm but is tried for comparison purposes and because it is not affected by any local minimum of the error function. For a more detailed discussion of the algorithms, please see the references cited. Before training the network, the input and output values are scaled based on the minimum and maximum values of the training data to prevent the saturation of the transfer functions of the PEs. The input values are scaled from -1.0 to 1.0. The output values are scaled from 0.2 to 0.8 when using the sigmoid transfer function or from -0.8 to 0.8 when using the hyperbolic tangent transfer function.

RESULTS AND DISCUSSION

Performance of learning algorithms: Preliminary computations using the three learning algorithms above show that NCDR learns fastest at the start of the learning process although the results are comparable with EDBD at the later part of the learning process (Figure 3). This may be attributed to the use of a smaller epoch size (the number of training examples presented to the network before updating the weights) for NCDR which results in more weight updates per one pass of the training set. The EDBD uses an epoch size equal to the training sample size. The DRS performed poorly mainly because of the large number of training examples. It is also noted that the choice of learning rates for the NCDR have a great effect on the learning curve especially at the later part of the learning process. Therefore, for subsequent computations, EDBD is used because the learning rates are adjusted automatically and the learning process is less sensitive to the selection of parameters.

For the three algorithms, the performance of the networks using the sigmoid transfer function is poor compared to the networks using the hyperbolic tangent transfer function.

Damage Estimation: Since the relationship between the strong ground motion indices and the ductility factor is complex and nonlinear, the network cannot predict the output precisely. The learning process, however, minimizes the errors associated with the training data. In this respect, it is similar to a multivariate regression, although an a priori functional form is not required. Figure 4 shows the distribution of the

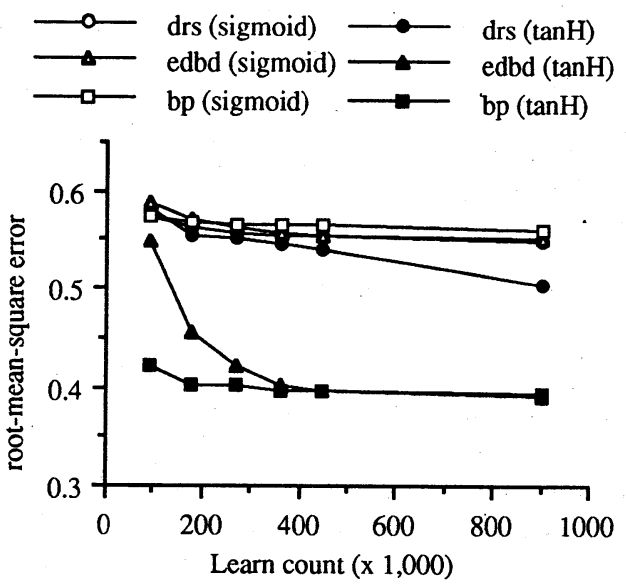

Figure 3. Performance curves of the three learning algorithms 
output based on the analysis vs. the network for the two structural models using EDBD.

Sensitivity Analysis: Although neural networks can find a relationship between the input and output values internally, it is not always easy to interpret the resulting weight state. Alternatively, it is possible to compute the sensitivity of the output value with respect to one of its input by taking the partial derivative. For a network with only one hidden layer having $M$ PEs and $N$ input PEs, the standard back-propagation equations for the output values of the hidden layer PEs and the outer layer PEs are

$$
\operatorname{out}_{j}^{h}=f_{h i}\left(\sum_{i=1}^{N} \omega_{j i}^{h} x_{i}+\theta_{j}^{h}\right) ; \quad \text { out }_{k}^{o}=f_{\text {oh }}\left(\sum_{j=1}^{M} \omega_{k j}^{o} \text { out }_{j}^{h}+\theta_{k}^{o}\right)
$$

where $\omega_{\mathrm{ji}}$ is the connection weight of the $j$ th PE from the $i$ th PE of the input layer, $\mathrm{x}_{\mathrm{i}}$ is the $i$ th scaled input, $\theta_{\mathrm{j}}$ is the bias term for the $j$ th $P E$ and $\mathrm{f}_{\text {hi }}$ and $\mathrm{f}_{\text {oh }}$ are the transfer functions. The superscripts define the variables for the outer layer and the hidden layer. The partial derivative of the output with respect to the input is then

$$
\begin{aligned}
\frac{\partial}{\partial x_{n}}\left(\text { out }_{k}^{o}\right)= & \dot{f}_{\text {oh }}\left(\sum_{j=1}^{M} \omega_{k j}^{o} \cdot f_{h i}\left(\sum_{i=1}^{N} \omega_{j i}^{h} x_{i}+\theta_{j}^{h}\right)+\theta_{k}^{o}\right) . \\
& \sum_{j=1}^{M}\left(\omega_{k j}^{o} \cdot \dot{f}_{h i}\left(\sum_{i=1}^{N} \omega_{j i}^{h} x_{i}+\theta_{j}^{h}\right) \cdot \omega_{j i}^{h}\right)
\end{aligned}
$$
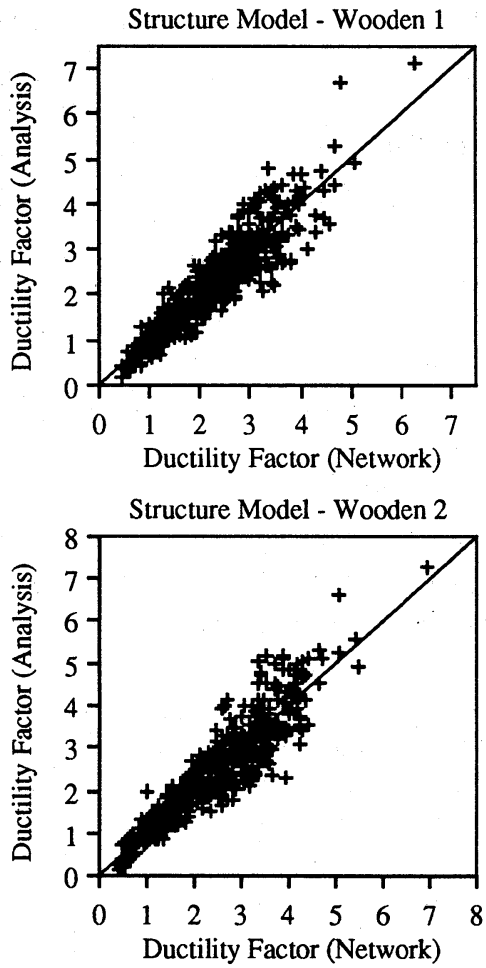

Figure 4. Distribution of Ductility Factor from analysis and from neural network

It can be seen from the above equation that the partial derivative depends not only on the weights and biases but also on the current values of the input variables, $\mathrm{x}_{\mathrm{i}^{*}}$. Thus it is difficult to generalize on the trend of the output value with respect to a change in a single input value. As an alternative, the distribution of the partial derivatives for the entire training set can be used to qualitatively describe the sensitivity of the output value. Figure 5 shows the histograms of the partial derivatives. It can be seen that the output is more sensitive to the PGA, SI, and root square and least sensitive to the time duration of motion.

Estimation using actual earthquakes: To test the performance of the network in actual earthquakes, the time history of a limited number of actual earthquakes is used. Two horizontal components of the El Centro
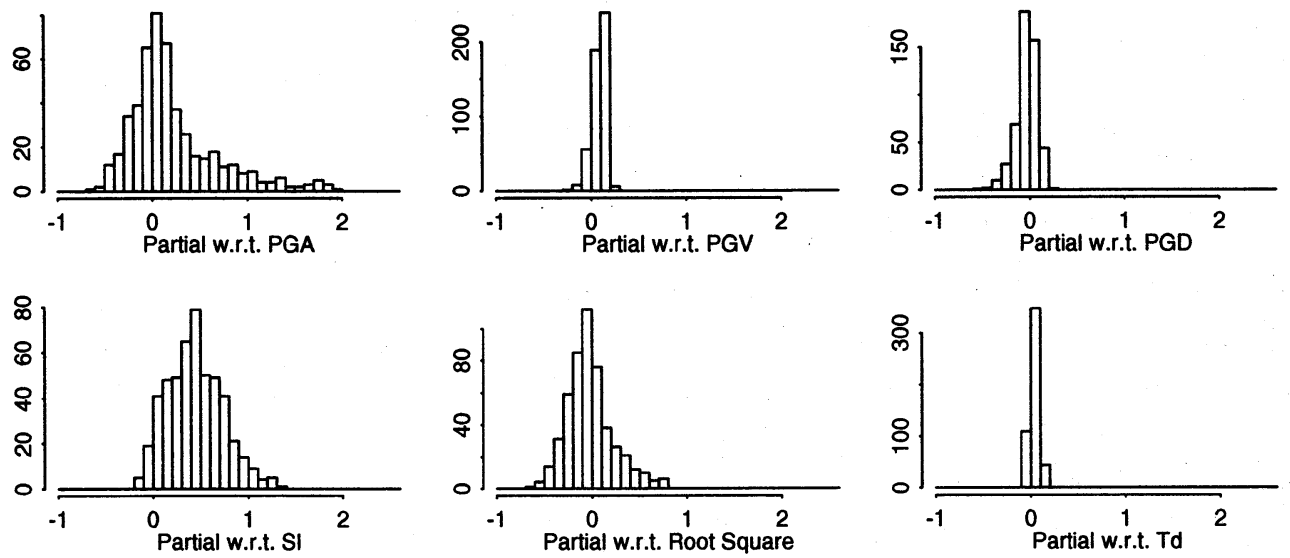

Figure 5. Distribution of partial derivatives of the scaled output with respect to the scaled input (for Wooden 1) 
Table 2. Ground motion indices of actual earthquakes

\begin{tabular}{|c|c|c|c|c|c|c|c|}
\hline EQ & dir & $\begin{array}{c}\text { PGA } \\
(\mathrm{cm} / \mathrm{s} / \mathrm{s})\end{array}$ & $\begin{array}{l}\text { PGV } \\
(\mathrm{cm} / \mathrm{s})\end{array}$ & $\begin{array}{l}\text { PGD } \\
(\mathrm{cm})\end{array}$ & $\begin{array}{c}\mathrm{SI} \\
(\mathrm{cm} / \mathrm{s})\end{array}$ & $\begin{array}{l}\text { root sqr } \\
\left(\mathrm{cm}^{2} / \mathrm{s}\right)^{-1 / 2}\end{array}$ & $\begin{array}{l}\mathrm{Td} \\
\text { (s) }\end{array}$ \\
\hline \multirow[t]{2}{*}{ El Centro } & NS & 340.2 & 34.3 & 7.8 & 39.0 & 330.2 & 24.4 \\
\hline & EW & 209.4 & 33.2 & 14.3 & 28.8 & 278.6 & 24.2 \\
\hline \multirow[t]{2}{*}{ Taft } & NS & 151.7 & 12.4 & 6.5 & 17.9 & 180.0 & 26.5 \\
\hline & EW & 176.0 & 14.9 & 5.2 & 19.3 & 187.8 & 25.7 \\
\hline \multirow[t]{2}{*}{ Niigata } & NS & 151.0 & 47.4 & 30.7 & 32.1 & 186.2 & 21.6 \\
\hline & EW & 156.5 & 52.9 & 21.8 & 30.5 & 161.6 & 24.5 \\
\hline Kushiro & NS & 816.9 & 67.3 & 9.3 & 75.2 & 1320.7 & 24.4 \\
\hline (KUS) & EW & 921.0 & 60.0 & 5.9 & 77.7 & 1474.1 & 19.0 \\
\hline Kushiro & NS & 196.5 & 11.0 & 2.7 & 13.8 & 200.0 & 18.1 \\
\hline (NEM) & EW & 216.4 & 9.6 & 2.1 & 12.1 & 213.1 & 17.4 \\
\hline
\end{tabular}

(1964), Taft (1952), Niigata (1964), and Kushiro(1993) earthquakes are used. For the Kushiro earthquake, two sets of acceleration time histories taken in two locations (JMA Kushiro and Nemuro stations) are used. Figure 6 shows the estimation of the neural networks for both structure models. The figure shows good prediction by the neural network except for two data points which belong to the time history at JMA Kushiro station for structure model Wooden 2. Figure 7 shows the response acceleration spectra for this time history. It can be seen that the structure Wooden 2 ( $\mathrm{T}=0.35 \mathrm{~s}$ ) will experience resonance while structure Wooden $1(\mathrm{~T}=0.55 \mathrm{~s})$ will have lower amplification. Therefore, it is recommended to include another input parameter that will detect the effect of resonance.

\section{CONCLUSIONS}

The use of neural networks for quick prediction of damage to specific structures from earthquake ground motion is demonstrated. To train the network, simulated ground motions are used to have a well-distributed training set. After training, the damage estimation of the neural network is found to be in good agreement with the analytical results in which the network is trained. A sensitivity analysis identifies that the peak ground acceleration, the Spectral Intensity, and the root square of the time history have the most effect on the results of the network while the time duration has the least effect. The network is used to estimate the damage using actual earthquakes and is found to give good results. However, poor results are obtained in one case where resonance of the structure is apparent. Therefore it is recommended that another input variable which can "identify" the resonance effect be added in training the network.

\section{References:}

1. M. Fatima, F. Yamazaki, and T. Katayama (1992). Neural networks for estimation of earthquake damage severity. Proc. 47th Annual Meeting JSCE, 142-143.

2. F.S. Wong, A.T.Y. Tung, and W. Dong (1992). Seismic hazard prediction using neural nets. Proc. 10th WCEE, 1, 339-343. 3. S.P. Lai (1982). Statistical characterization of strong ground motions using power spectral density function. BSSA. 72:1, 259274.

4. T. Katayama, N. Sato, and K. Saito (1988). SI sensors for the identification of destructive earthquake ground motion. Proc.9th WCEE.

5. M.D. Trifunac and A.G. Brady (1973). A study of the duration of strong earthquake ground motion. BSSA. 65.

6. J.L. McClelland and D.E. Rumelhart (1988). Explorations in Parallel Distributed Processing (A handbook of models, programs and exercises). MIT Press

7. A.A. Minai and R.D. Williams (1990). Acceleration of back-propagation through learning rate and Momentum Adaptation. Intn'l Joint Conf. on Neural Networks. 1. 676-679.

8. N. Baba (1989). A new approach for finding the global minimum of error function of neural networks. Neural Networks. 2. 246-253. 